\title{
Research Article \\ Optimal Control for Multistage Nonlinear Dynamic System of Microbial Bioconversion in Batch Culture
}

\author{
Lei Wang, ${ }^{1,2}$ Zhilong Xiu, ${ }^{1}$ Yuduo Zhang, ${ }^{2}$ and Enmin Feng ${ }^{2}$ \\ ${ }^{1}$ School of Life Science and Biotechnology, Dalian University of Technology, Dalian, \\ Liaoning 116012, China \\ 2 School of Mathematical Science, Dalian University of Technology, Dalian, Liaoning 116024, China \\ Correspondence should be addressed to Lei Wang,wanglei@dlut.edu.cn
}

Received 30 April 2011; Revised 23 May 2011; Accepted 3 June 2011

Academic Editor: Kuppalapalle Vajravelu

Copyright (C) 2011 Lei Wang et al. This is an open access article distributed under the Creative Commons Attribution License, which permits unrestricted use, distribution, and reproduction in any medium, provided the original work is properly cited.

\begin{abstract}
In batch culture of glycerol biodissimilation to 1,3-propanediol (1,3-PD), the aim of adding glycerol is to obtain as much 1,3-PD as possible. Taking the yield intensity of 1,3-PD as the performance index and the initial concentration of biomass, glycerol, and terminal time as the control vector, we propose an optimal control model subject to a multistage nonlinear dynamical system and constraints of continuous state. A computational approach is constructed to seek the solution of the above model. Firstly, we transform the optimal control problem into the one with fixed terminal time. Secondly, we transcribe the optimal control model into an unconstrained one based on the penalty functions and an extension of the state space. Finally, by approximating the control function with simple functions, we transform the unconstrained optimal control problem into a sequence of nonlinear programming problems, which can be solved using gradient-based optimization techniques. The convergence analysis and optimality function of the algorithm are also investigated. Numerical results show that, by employing the optimal control, the concentration of 1,3-PD at the terminal time can be increased, compared with the previous results.
\end{abstract}

\section{Introduction}

The bioconversion of glycerol to 1,3-propanediol (1,3-PD) has recently received more and more attention throughout the world due to its environmental safety, high region specificity, cheaply available feedstock, and relatively high theoretical molar yield [1]. Many researchs have been carried out including the quantitative description of the cell growth kinetics of multiple inhibitions, the metabolic overflow kinetics of substrate consumption and product formation [2-4], open-loop substrate input and $\mathrm{pH}$ logic control [5], enzyme-catalytic reductive pathway and transport of glycerol and 1,3-propanediol across cell membrane [6], parameter identification of biochemical systems [7] and feedback control and pulse feeding 
[8] for the models of the continuous cultures, feeding strategy of glycerol [9], and optimal control [10] and optimality condition [11] in fed-batch culture.

Compared with continuous and feed-batch cultures, glycerol fermentation in batch culture can obtain the highest production concentration and molar yield 1,3-PD to glycerol [12]. So nonlinear dynamical systems in this culture have been extensively considered in recent years [13-15]. In batch culture of glycerol biodissimilation to 1,3-propanediol (1,3PD), the aim of adding glycerol is to obtain as much 1,3-PD as possible. In this paper, based on the previous model in [16], taking the yield intensity of 1,3-PD as the performance index and the initial concentration of biomass, glycerol and terminal time as the control vector, we propose an optimal control model subject to a multistage nonlinear dynamical system and constraints of continuous state. A computational approach is constructed to seek the solution of the above model in two aspects. On the one hand transform the optimal control problem into the one with fixed terminal time and transcribe it into an unconstrained one based on the penalty functions and an extension of the state space; on the other hand, by approximating the control function with simple functions, we transform the unconstrained optimal control problem into a sequence of nonlinear programming problems, which can be solved using gradient-based optimization techniques. The convergence analysis and optimality function of the algorithm are also investigated. Numerical results show that, by employing the optimal control, the concentration of 1,3-PD at the terminal time can be increased, compared with the previous results.

This paper is organized as follows. In Section 2, a nonlinear dynamical system of batch culture is proposed. In Section 3, we propose an optimal control model, develop a computational approach to solve the optimal control model, and prove the convergence of algorithm. Section 4 illustrates the numerical results. Finally, conclusions are provided in Section 5.

\section{Nonlinear Dynamical System}

On the basis of our previous literature(see [16]), mass balances of biomass, substrate, and products in batch culture can be formulated as the following nonlinear dynamical system:

$$
\begin{gathered}
\dot{x}(t)=f(t, x(t)), \quad t \in\left[0, t_{f}\right], x(0)=\xi, \\
f(t, x(t))=\left(f_{1}(t, x(t)), f_{2}(t, x(t)), f_{3}(t, x(t)), f_{4}(t, x(t)), f_{5}(t, x(t))\right)^{T} \\
=\left(\mu x_{1}(t),-q_{2} x_{1}(t), q_{3} x_{1}(t), q_{4} x_{1}(t), q_{5} x_{1}(t)\right)^{T},
\end{gathered}
$$

where $x_{1}(t), x_{2}(t), x_{3}(t), x_{4}(t)$, and $x_{5}(t)$ are biomass, glycerol, 1,3-PD, acetate, and ethanol concentrations at time $t$ in the reactor, respectively. $\xi=\left(\xi_{1}, \xi_{2}, \xi_{3}, \xi_{4}, \xi_{5}\right)^{T}$ denotes the initial state, and $t_{f}$ is the terminal time of the fermentation process. $x \triangleq\left(x_{1}, x_{2}, x_{3}, x_{4}, x_{5}\right)^{T} \in R_{+}^{5}$ is as state vector. The specific growth rate of cells $\mu$, specific consumption rate of substrate, $q_{2}$ 
and specific formation rate of products $q_{i}, i=3,4,5$, are expressed by the following equations on the basis of $[13,16]$ :

$$
\begin{gathered}
\mu=\mu_{m} \exp \left(\frac{-\left(t-t_{m}\right)^{2}}{2 t_{l}^{2}}\right) \prod_{i=2}^{5}\left(1-\frac{x_{i}}{x_{i}^{*}}\right), \\
q_{2}=m_{2}+\frac{\mu}{Y_{2}} \\
q_{i}=m_{i}+\mu Y_{i}, \quad i=3,4,5 .
\end{gathered}
$$

In batch culture, the initial concentrations of biomass, glycerol, and the terminal time can be chosen as control variables. Let $u=\left(u_{1}, u_{2}, u_{3}\right)^{T} \triangleq\left(\xi_{1}, \xi_{2}, t_{f}\right)^{T} \in R_{+}^{3}$ be the control vector. The solution of system (2.1) with respect to control vector is defined by $x(\cdot, u)$.

Based on the factual fermentation, there exist critical concentrations, outside which cells cease to grow, of biomass, glycerol, 1,3-PD, acetate and ethanol. Hence, it is biologically meaningful to restrict the concentrations of biomass, glycerol, products, and the volume of culture fluid in a set $W$ and the control vector in a admissible control set $U$ defined respectively, as follows:

$$
\begin{gathered}
x(t, u) \in W \triangleq \prod_{i=1}^{5}\left[x_{* i}, x_{i}^{*}\right] \subset R_{+}^{5}, \quad \forall t \in I=\left[0, t_{f}\right] . \\
u \in U \triangleq \prod_{i=1}^{3}\left[u_{* i}, u_{i}^{*}\right] \subset R_{+}^{3} .
\end{gathered}
$$

Let $C_{b}\left([0, T], R^{5}\right)$ denote the space of continuous bounded functions on $[0, T]$ with values in $R^{5}$, equipped with the sup-norm topology, that is, for $z \in C_{b}\left([0, T], R^{5}\right),\|z\|_{c}=$ $\sup \{\|z(t)\|, t \in[0, T]\}$, where $\|\cdot\|$ is the Euclidean norm.

\section{Optimal Control Problem}

The optimal control problem using the yield intensity of 1,3-PD at the terminal time as cost functional, based on the controlled multistage nonlinear dynamical system (2.1), can be formulated as follows:

$$
\begin{array}{ll}
\text { inf } & J(u) \triangleq-\frac{x_{3}\left(u_{3}, u\right)}{u_{3}} \\
\text { s.t. } & \dot{x}(t)=f(t, x(t, u)) \\
& x(0)=\xi, \\
& x(t) \in W, \quad t \in\left[0, u_{3}\right] \\
& u \in U .
\end{array}
$$

From the theory on continuous dependence of solutions on parameters and our previous literature (see [16]), we know that $x(\cdot, u)$ is continuous relative to $u$, so $J(u)$ is 
continuous on $u \in U$. Moreover, $U$ is a closed bounded convex subset of $R_{+}^{3}$. Hence we know the optimal control must exist, namely, $\exists u^{*} \in U$ such that $J\left(u^{*}\right) \leq J(u)$, for all $u \in U$.

\subsection{Differentiability with respect to the Control Vector}

In this subsection, our aim is to show the differentiability and the gradient information of solutions of the system (2.1) with respect to the control vector. To begin with, we discuss some properties of the function $f(t, x(t, u))$.

Proposition 3.1. For the system (2.1), $f(t, x(t, u))$ and $\left(\partial f / \partial x_{i}\right)(t, x(t, u))(i=1,2, \ldots, 5)$ are continuous in $(t, x)$ on an open set $\Delta$ in $R_{+} \times W$.

Proof. It follows by inspection that the function $f \in C^{2}\left(R_{+} \times W, R^{5}\right)$ by definition and (2.3).

Using Theorems I-1-4 and II-1-2 in [17], we can show that the system (2.1) has a unique solution $x=x(t, u)$, and the solution satisfies the integral equation

$$
x(t, u)=\xi+\int_{0}^{t} f(s, x(s, u)) d s, \quad t \in I
$$

$u_{1}=\xi_{1}$ and $u_{2}=\xi_{2}$ are the initial value of the system (2.1), due to the differentiability of $x(t, u)$ with respect to the initial vector, and we have

$$
\frac{\partial f}{\partial \xi_{j}}(t, x(t, \xi, u))=e_{j}+\int_{0}^{t} \frac{\partial f}{\partial x}(s, x(t, \xi, u)) \frac{\partial x}{\partial \xi_{j}}(s, x(t, \xi, u)) d s,
$$

where $e_{j}$ is the vector in $R^{5}$ with entries 0 except for 1 at the $j$ th entry, and $\partial f / \partial x$ is the $5 \times 5$ matrix whose $i$ th column is $\partial f / \partial x_{i}, i, j=1,2, \ldots, 5$. From this speculation, we obtain the following result.

Proposition 3.2. Partial derivatives $\partial x / \partial u_{1}$ and $\partial x / \partial u_{2}$ exist and are continuous in $(t, u)$. Furthermore, $\partial x / \partial \xi_{j}$ is the unique solution of the initial-value problem

$$
\dot{z}=\frac{\partial f}{\partial x}(t, x(t, u)) z, \quad z(0)=-e_{j} .
$$

By virtue of the result of Proposition 3.2, we can obtain the value of $\partial x / \partial u_{1}$ and $\partial x / \partial u_{2}$. The following Proposition gives a formula to compute the value of $\partial x / \partial u_{3}$.

Proposition 3.3. Partial derivatives $\partial x / \partial u_{3}$ exist and are continuous in $(t, u)$. Furthermore,

$$
\frac{\partial x}{\partial u_{3}}=f\left(u_{3}, x\left(u_{3}, u\right)\right) .
$$


Proof. The existence and continuity of $\partial x / \partial u_{3}$ can be directly obtained by the function $f \in$ $C^{2}\left(R_{+} \times W, R^{5}\right)$ and implicit function theorem. Next, we derive the formula (3.4), for $\forall t \in I$, and we have:

$$
\begin{gathered}
x\left(u_{3}, u\right)=\xi+\int_{0}^{u_{3}} f(s, x(s, u)) d s, \\
x\left(u_{3}+\Delta t, u\right)=\xi+\int_{0}^{u_{3}+\Delta t} f(s, x(s, u)) d s,
\end{gathered}
$$

then, by the integral mean value theorem, there exists a constant $\theta \in[0,1]$, such that

$$
x\left(u_{3}+\Delta t, u\right)-x\left(u_{3}, u\right)=\int_{u_{3}}^{u_{3}+\Delta t} f(s, x(s, u)) d s=f\left(u_{3}+\theta \Delta t, x\left(u_{3}+\theta \Delta t, u\right)\right) \Delta t .
$$

Let $\Delta t \rightarrow 0$, then

$$
\frac{\partial x}{\partial u_{3}}=\lim _{\Delta t \rightarrow 0} \frac{x\left(u_{3}+\Delta t, u\right)-x\left(u_{3}, u\right)}{\Delta t}=\lim _{\Delta t \rightarrow 0} f\left(u_{3}+\theta \Delta t, x\left(u_{3}+\theta \Delta t, u\right)\right)=f\left(u_{3}, x\left(u_{3}, u\right)\right) .
$$

We obtain the desired result.

\subsection{Model Transformation}

The optimal control problem (3.5) is not a standard case because the terminal time $t_{f}$ is free. Using the method in Section 6.8.1 of [18], the (3.5) can be transformed into the one with fixed terminal time. Treating $t_{f}$ as an unknown parameter and using the transformation $t=t_{f} \tau$, the (3.5) is converted to (3.8) as follows:

$$
\begin{array}{ll}
\inf & J(u) \triangleq-\frac{x_{3}(1, u)}{u_{3}} \\
\text { s.t. } & \dot{x}(\tau)=t_{f} f\left(t_{f} \tau, x\left(t_{f} \tau, u\right)\right) \\
& x(0)=\xi, \\
& x(\tau) \in W, \quad \tau \in[0,1] \\
& u \in U .
\end{array}
$$




\subsection{Semi-Infinite Optimization with Inequality Constrained}

For the optimal control problem $J(u)$, it is difficult to cope with the continuous state inequality constraints, that is, this is a semi-infinite optimization problem. To overcome the difficulty, let

$$
\begin{gathered}
g_{i}(x(\tau, u)):=x_{i}(\tau, u)-x_{i}^{*} \\
g_{5+i}(x(\tau, u)):=x_{* i}-x_{i}(\tau, u), \quad i=1,2, \ldots, 5 .
\end{gathered}
$$

The condition, $x(\tau, u) \in W$, for all $\tau \in[0,1]$, is equivalently transcribed into

$$
G(u)=0,
$$

where $G(u)=\sum_{l=1}^{10} \int_{0}^{1} \max \left\{0, g_{i}(x(\tau, u))\right\} d t$.

However, $G(u)$ is nonsmooth in $g_{i}(x(\tau, u))=0$. By the standard optimization routines $[19,20]$, the following smoothing technique is to replace $\widehat{g}_{i, \epsilon}(x(\tau, u))$ with $\max \left\{0, g_{i}(x(\tau, u))\right\}$, where

$$
\widehat{g}_{i, \epsilon}(x(\tau, u))= \begin{cases}0 & \text { if } g_{i}(x(\tau, u))<-\epsilon, \\ \frac{\left(g_{i}(x(\tau, u))+\epsilon\right)^{2}}{4 \epsilon} & \text { if }-\epsilon \leq g_{i}(x(\tau, u)) \leq \epsilon \\ g_{i}(x(\tau, u)) & \text { if } g_{i}(x(\tau, u))>\epsilon .\end{cases}
$$

Note that

$$
G_{\epsilon}(u)=\sum_{l=1}^{10} \int_{0}^{T} \widehat{g}_{l, \epsilon}(x(\tau, u)) d t
$$

is a smooth function in $u$. Let

$$
\begin{aligned}
W_{\epsilon} & \triangleq\left\{u \in U \mid G_{\epsilon}(u)=0\right\} \\
& =\left\{u \in U \mid g_{l}(x(\tau, u)) \leq-\epsilon, l=1,2, \ldots, 10, \tau \in[0,1]\right\} .
\end{aligned}
$$

Clearly, $W_{\epsilon} \subseteq W \cap U$ for each $\epsilon>0$. 
We now define an approximate problem denoted by (3.13), where the smoothed state constraint is treated as the penalty function:

$$
\begin{array}{ll}
\inf & J_{\epsilon, r}(u):=-x_{3}(1, u)+r G_{\epsilon}(u) \\
\text { s.t. } & \dot{x}(t)=t_{f} f\left(t_{f} \tau, x\left(t_{f} \tau, u\right)\right), \\
& x(0)=\xi \\
& u \in W_{\epsilon} .
\end{array}
$$

By similar arguments as those given in [21], (3.13) is coincident with (3.5) as $\epsilon \rightarrow 0$. On this basis, (3.5) can be explored by solving a sequence of approximate (3.13). Each of these (3.13) is viewed as a smooth nonlinear mathematical programming problem.

\subsection{Optimization Algorithm}

In this subsection, similar with the approach based on gradient in [22, 23], we proposed an algorithm based on gradient of $J_{\epsilon, \gamma}(u)$ to solve the (3.13). $\partial J_{\epsilon, \gamma}(u) / \partial u \triangleq J_{g}(u)$ can be derived by solving the ordinary differential equation (3.3) and computing (3.4). The admissible control set $U$ can be called a "box" because of its rectangular shape, we use the classic gradient projection method to cope with "box."

The projection of an arbitrary $u$ onto the feasible set $U$ is defined as follows. The $i$ th component is given by

$$
p\left(u, u_{*}, u^{*}\right)_{i}= \begin{cases}u_{i *} & \text { if } u_{i}<u_{i * \prime} \\ u_{i} & \text { if } u_{i} \in\left[u_{i *}, u_{i}^{*}\right] \\ u_{i}^{*} & \text { if } u_{i}>u_{i}^{*}\end{cases}
$$

Thus, the control variable $u(t)$ obtained by projecting the steepest descent direction at $u$ onto the feasible set $U$ is given by

$$
u(\lambda)=p\left(u-\lambda J_{g}, u_{*}, u^{*}\right)
$$

where $\lambda$ is optional step size.

On the basis of the above analysis, we can obtain an optimal control for (3.13) as shown in the following algorithm.

\section{Algorithm 3.4.}

Step 1. Set constants $\alpha, \delta \in(0,1)$, and $r_{\max }$ is positive constant. Set $r=0$, compute $J_{g}\left(u_{r}\right)$ by equations (3.3) and (3.4), if $J_{g}\left(u_{r}\right)<\delta$, stop. Else, then go to Step 2. 
Step 2. Compute the step-size $\lambda(r)=\beta^{k_{r}}$ with Armijo line search rules, where $k_{r}$ is any integer such that

$$
\begin{aligned}
& J_{\epsilon, r}\left(u(r)+\beta^{k_{r}} J_{g}\left(u_{r}\right)\right)-J_{\epsilon, \gamma}(u(r)) \leq-\beta^{k_{r}} \alpha J_{g}^{T}\left(u_{r}\right) J_{g}\left(u_{r}\right), \\
& J_{\epsilon, r}\left(u(r)+\beta^{k_{r}} J_{g}\left(u_{r}\right)\right)-J_{\epsilon, r}(u(r))>-\beta^{k_{r-1}} \alpha J_{g}^{T}\left(u_{r}\right) J_{g}\left(u_{r}\right),
\end{aligned}
$$

go to Step 3.

Step 3. If $r>r_{\max }$, stop. Otherwise, compute $u(r+1)=p\left(u(r)-\lambda(r) J_{g}\left(u_{r}\right), u_{*}, u^{*}\right)$ using the equation defined by (3.14), replace $r$ by $r+1$, and go to Step 1 .

Note that, due to the boundedness of the function $J_{\epsilon, r}(\cdot)$, it is very easy to find a $k_{r}$ satisfying (3.15) and (3.16), using the following subprocedure, which uses the last used step length $\lambda_{r-1}=\beta^{k_{r-1}}$, as the starting point for the computation of the next one.

\section{Subprocedure of Algorithm 3.4}

Step 1. If $r=0$, set $k^{\prime}=0$. Else, set $k^{\prime}=k_{r-1}$.

Step 2. If $k_{r}=k^{\prime}$ satisfies (3.15) and (3.16). Else, set $k^{\prime}=k_{r-1}$, stop.

Step 3. If $k_{r}=k^{\prime}$ satisfies (3.15) and not (3.16), replace $k^{\prime}$ by $k^{\prime}-1$, and go to Step 2. If $k_{r}=k^{\prime}$ satisfies (3.16) and not (3.15), replace $k^{\prime}$ by $k^{\prime}+1$, and go to Step 2 .

For Algorithm 3.4, we see that $-J_{g}^{T} J_{g}(\cdot)$ is continuous, that $-J_{g}^{T} J_{g}(u) \leq 0$ for all $u \in U$, and that $-J_{g}^{2}(u)=0$ if and only if $J_{g}(u)=0$, that is, that $-J_{g}^{2}(\cdot)$ is an optimality function for the problem (3.13). So, we have the following theorem to guarantee the convergence of the algorithm.

Theorem 3.5. If $u_{r}$ is such that $J_{g} \neq 0$, then $\lambda_{r}$ is computed by Algorithm 3.4 using a finite number of function evaluations and any accumulation point $\widehat{u}$ of this sequence satisfies $J_{g}(\widehat{u})=0$.

Proof. We apply Theorem 1.2.24a in [24] with $\theta(\cdot)=-J_{g}^{T} J_{g}(\cdot)$, then the desired result can be obtained immediately.

Remark 3.6. Although the approach we are using here to deal with the constraints of continuous state is similar with the one mentioned in [19], There are still three main difference between them. First of all, our approach is applied for the batch culture, while the one in [19] is used for the fed-batch culture. Secondly, the control variables in the two approaches are different. Our variable controls the initial and terminal points, and their variable controls the switching time. Finally, we use the gradient-based algorithm to numerically solve the problem. Their algorithm is an improved Particle Swarm Optimization (PSO) algorithm, not gradient based. 
Table 1: Parameters values in dynamical system (2.1).

\begin{tabular}{lccccc}
\hline Substrate/products & $t_{l}$ & $t_{m}$ & $\mu_{m}$ & $m_{i}$ & $Y_{i}$ \\
\hline$i=1$ (Biomass) & 1.7924 & 2.4508 & 0.9192 & - & - \\
$i=2$ (Glycerol) & - & - & - & 1.358 & 0.01558 \\
$i=3$ (1,3-PD) & - & - & - & -8.9346 & 64.69 \\
Acetic acid & - & - & - & 2.1098 & 4.541 \\
Ethanol & - & - & - & -0.183 & 3.046 \\
\hline
\end{tabular}

\section{Numerical Results}

According to the model and algorithm mentioned above, we have programmed the software and applied it to the optimal control problem of microbial fermentation in batch culture. The system parameters are listed in Table 1 (see $[9,16])$.

The basic data are listed, respectively, as follows.

\section{Boundary Value of Control Vector}

$u_{* 1}=0.01 \mathrm{mmol} / \mathrm{L}, u_{1}^{*}=1 \mathrm{mmol} / \mathrm{L}, u_{* 2}=200 \mathrm{mmol} / \mathrm{L}, u_{2}^{*}=939.5 \mathrm{mmol} / \mathrm{L}, u_{* 3}=2 h$, and $u_{3}^{*}=10 \mathrm{~h}$.

\section{Boundary Value of State Vector}

$x_{* 1}=0.001 \mathrm{mmol} / \mathrm{L}, x_{1}^{*}=2039 \mathrm{mmol} / \mathrm{L}, x_{* 2}=0.001 \mathrm{mmol} / \mathrm{L}, x_{2}^{*}=939.5 \mathrm{mmol} / \mathrm{L}, x_{* 3}=$ $0.01 \mathrm{mmol} / \mathrm{L}, x_{3}^{*}=10 \mathrm{mmol} / \mathrm{L} . u_{* 4}=0.01 \mathrm{mmol} / \mathrm{L}, u_{4}^{*}=1026 \mathrm{mmol} / \mathrm{L}$, and $u_{* 5}=200, u_{5}^{*}=$ $360.9 \mathrm{mmol} / \mathrm{L}$.

We adopt $\alpha=0.4, \delta=0.00001$, and $r_{\max }=1000$ in the procedure. Then, by Algorithm 3.4, the optimal control vector $\bar{u}$ and objective function $J_{\epsilon, r}(\bar{u})$ are $(0.973186,547.04,5.17509)^{T}$ and 54.5911, respectively. Numerical results show that, by employing the optimal control, the concentration of 1,3-PD at the terminal time can be increased, compared with the previous results.

\section{Conclusions}

In this paper, based on the previous model in [16], taking the yield intensity of 1,3-PD as the performance index and the initial concentration of biomass, glycerol, and terminal time as the control vector, we propose an optimal control model subject to a multistage nonlinear dynamical system and constraints of continuous state. A computational approach is constructed to seek the solution of the above model in two aspects. The convergence analysis and optimality function of the algorithm are also investigated. Numerical results show that, by employing the optimal control, yield intensity of 1,3-PD at the terminal time can be increased, compared with the previous results.

Our current tasks accommodate the modeling and simulation of the fermentation process. Moreover, the stability and reachability of the improved model need to be discussed. 


\section{Acknowledgments}

This work was supported by the National Natural Science Foundation of China (no. 10871033), the Natural Science Foundation for the Youth of China (no. 11001153) the Shandong Province Natural Science Foundation of China (no. ZR2010AQ016) and the Fundamental Research Funds for the Central Universities.

\section{References}

[1] A. P. Zeng, H. Biebl, and H. Schlieker, "Pathway analysis of glycerol fermentation by K. pneumoniae: regulation of reducing equivalent balance and product formation," Enzyme and Microbial Technology, vol. 15, pp. 770-779, 1993.

[2] Z. L. Xiu, "Research progress on the production of 1,3-propanediol by fermentation," Microbiology, vol. 27, pp. 300-302, 2000.

[3] A. P. Zeng and H. Biebl, "Bulk chemicals from biotechnology: the case of 1,3-propanediol production and the new trends," Advances in Biochemical Engineering/Biotechnology, vol. 74, pp. 239-259, 2002.

[4] A. P. Zeng, K. Menzel, and W. D. Deckwer, "Kinetic, dynamic, and pathway studies of glycerol metabolism by Klebsiella pneumoniae in anaerobic continuous culture: II. Analysis of metabolic rates and pathways under oscillation and steady-state conditions," Biotechnology and Bioengineering, vol. 52, pp. 561-571, 1996.

[5] J. X. Ye, E. M. Feng, H. C. Yin, and Z. L. Xiu, "Modelling and well-posedness of a nonlinear hybrid system in fed-batch production of 1,3-propanediol with open loop glycerol input and $\mathrm{pH}$ logic control," Nonlinear Analysis. Real World Applications, vol. 12, no. 1, pp. 364-376, 2011.

[6] Y. Q. Sun, W. T. Qi, H. Teng, Z. L. Xiu, and A. P. Zeng, "Mathematical modeling of glycerol fermentation by Klebsiella pneumoniae: concerning enzyme-catalytic reductive pathway and transport of glycerol and 1,3-propanediol across cell membrane," Biochemical Engineering Journal, vol. 38, no. 1, pp. 22-32, 2008.

[7] L. Wang, Z. L. Xiu, Z. H. Gong, and E. M. Feng, “Modeling and parameter identification for multistage simulation of microbial bioconversion in batch culture," International Journal of Biomathematics. In press.

[8] Y. Tian, K. B. Sun, A. Kasperski, and L. S. Chen, "Nonlinear modelling and qualitative analysis of a real chemostat with pulse feeding," Discrete Dynamics in Nature and Society, vol. 2010, Article ID 640594, 18 pages, 2010.

[9] C. Gao, E. M. Feng, Z. T. Wang, and Z. L. Xiu, "Nonlinear dynamical systems of bio-dissimilation of glycerol to 1,3-propanediol and their optimal controls," Journal of Industrial and Management Optimization, vol. 1, no. 3, pp. 377-388, 2005.

[10] G. Wang, E. M. Feng, and Z. L. Xiu, "Vector measure as controls for explicit nonlinear impulsive system of fed-batch culture," Journal of Mathematical Analysis and Applications, vol. 351, no. 1, pp. 120 127, 2009.

[11] H. Y. Wang, E. M. Feng, and Z. L. Xiu, "Optimality condition of the nonlinear impulsive system in fed-batch fermentation," Nonlinear Analysis. Theory, Methods E Applications, vol. 68, no. 1, pp. 12-23, 2008.

[12] B. Gtinzel, Mikrobielle herstellung von 1,3-propandiol durch clostridium butyricum und adsorptive abtremutng von diolen, Ph.D. thesis, TU Braunschweig, Germany, 1991.

[13] C. Gao et al., "Parameter identification and optimization of process for bio-dissimilation of glycerol to 1, 3-propanediol in batch culture," Journal of Dalian University of Technology, vol. 46, no. 5, pp. 771-774, 2006.

[14] X. H. Li, E. M. Feng, and Z. L. Xiu, “Optimal control and property of nonlinear dynamic system for microorganism in batch culture," OR Transaction, vol. 9, no. 4, pp. 67-79, 2005.

[15] J. Wang, J. X. Ye, E. M. Feng, H. C. Yin, and Z. L. Xiu, "Modeling and identification of a nonlinear hybrid dynamical system in batch fermentation of glycerol," Mathematical and Computer Modelling, vol. 54, no. 1-2, pp. 618-624, 2011.

[16] L. Wang, J. X. Ye, E. M. Feng, and Z. L. Xiu, “An improved model for multistage simulation of glycerol fermentation in batch culture and its parameter identification," Nonlinear Analysis. Hybrid Systems, vol. 3, no. 4, pp. 455-462, 2009. 
[17] P.-F. Hsieh and Y. Sibuya, Basic Theory of Ordinary Differential Equations, Springer, New York, NY, USA, 1999.

[18] K. L. Teo, C. J. Goh, and K. H. Wong, A Unified Computational Approach to Optimal Control Problems, vol. 55, Longman Scientific \& Technical, Harlow, UK, 1991.

[19] C. Y. Liu, Zhaohua Gong, and E. M. Feng, "Modelling and optimal control for nonlinear multistage dynamical system of microbial fed-batch culture," Journal of Industrial and Management Optimization, vol. 5, no. 4, pp. 835-850, 2009.

[20] C. Y. Liu and E. M. Feng, "Optimal control of switched autonomous systems in microbial fed-batch cultures," International Journal of Computer Mathematics, vol. 88, no. 2, pp. 396-407, 2011.

[21] K. L. Teo, V. Rehbock, and L. S. Jennings, "A new computational algorithm for functional inequality constrained optimization problems," Automatica, vol. 29, no. 3, pp. 789-792, 1993.

[22] Q. Lin, R. C. Loxton, K. L. Teo, and Y. H. Wu, “A new computational method for optimizing nonlinear impulsive systems," Dynamics of Continuous, Discrete $\mathcal{E}$ Impulsive Systems. Series B, vol. 18, no. 1, pp. 59-76, 2011.

[23] R. C. Loxton, K. L. Teo, and V. Rehbock, "Optimal control problems with multiple characteristic time points in the objective and constraints," Automatica, vol. 44, no. 11, pp. 2923-2929, 2008.

[24] E. Polak, Optimization, vol. 124 of Applied Mathematical Sciences, Springer, New York, NY, USA, 1997. 


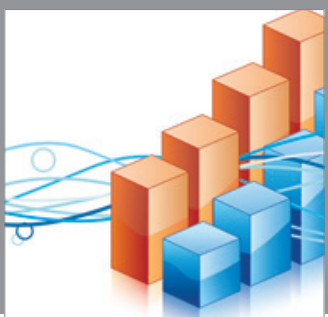

Advances in

Operations Research

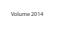

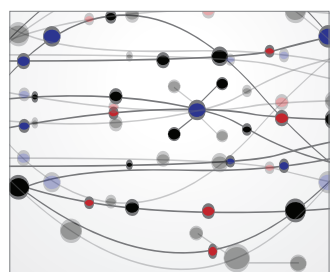

\section{The Scientific} World Journal
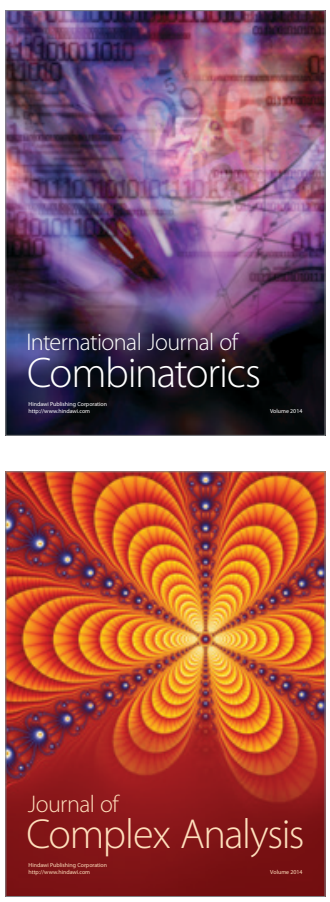

International Journal of

Mathematics and

Mathematical

Sciences
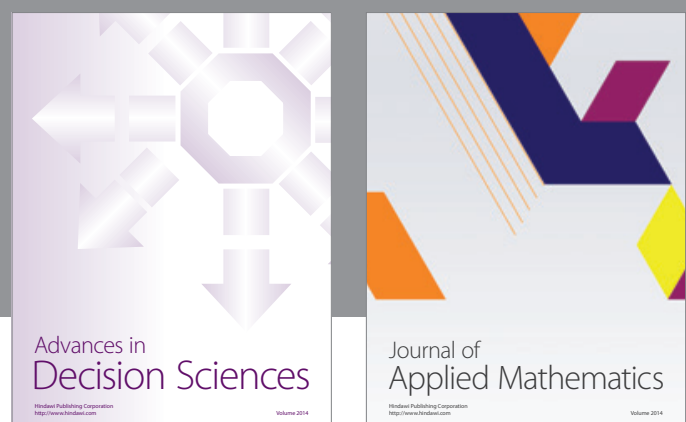

Journal of

Applied Mathematics
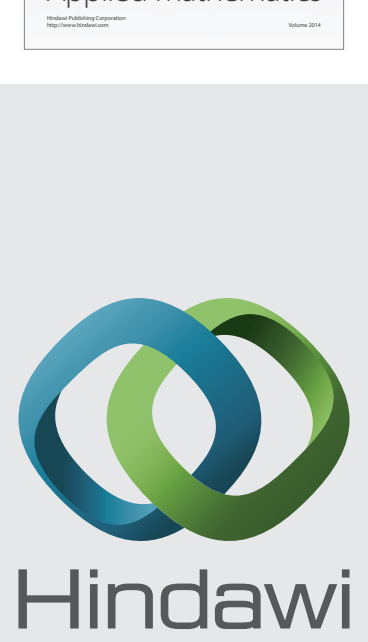

Submit your manuscripts at http://www.hindawi.com
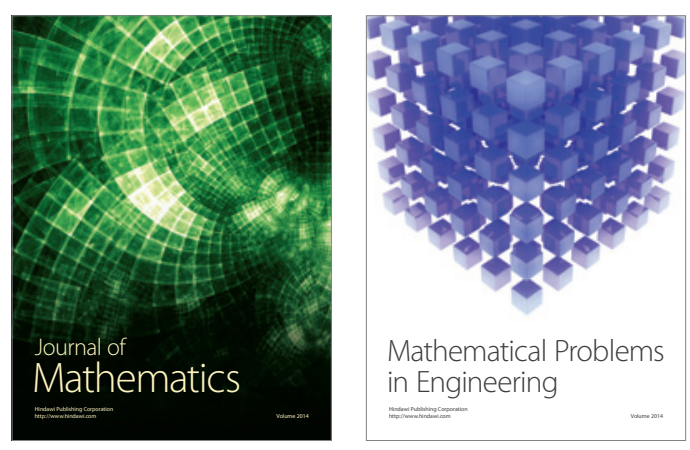

Mathematical Problems in Engineering
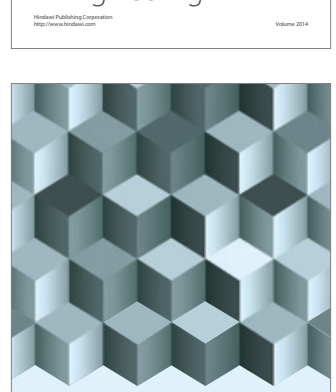

Journal of

Function Spaces
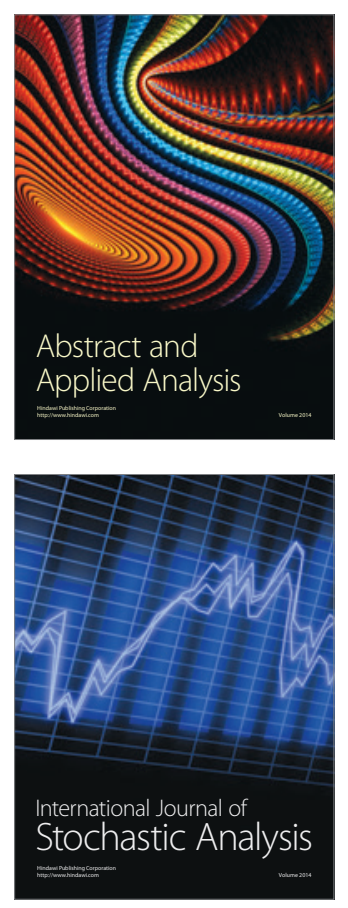

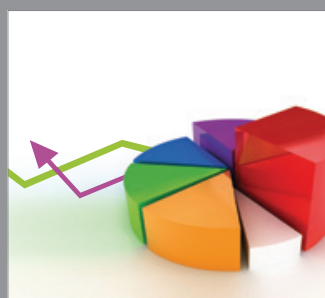

ournal of

Probability and Statistics

Promensencen
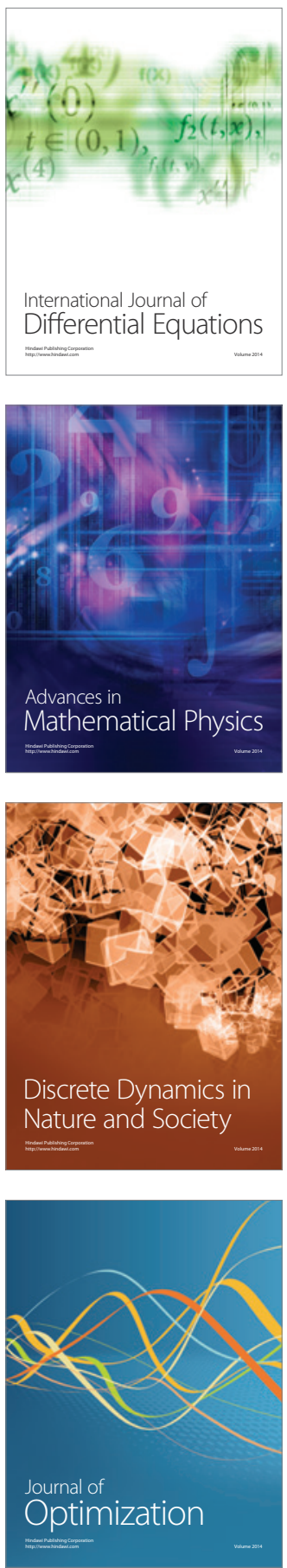